\title{
ESTUDIO COMPARATIVO ENTRE LA ACCION DE UN NUEVO AMINO-GLUCOSIDO (NETILMICINA) Y LA DE NUEVE ANTIBIOTICOS DE USO COMUN SOBRE CEPAS BACTERIANAS COLOMBIANAS
}

\author{
ERIX BOZON, ${ }^{*}$ MIGUEL GUZMAN, ${ }^{\star \star}$ MERCY DE GUEVARA, ${ }^{\star \star \star}$ ALVARO AGUILERA. ${ }^{* \star \star \star}$
}

\begin{abstract}
Se hace un estudio descriptivo sobre el comportamiento de cepas bacterianas de diez y siete microorganismos frente a diez antibibticos de amplio espectro, de uso más común en nuestro medio, dentro de los cuales se encuentra un aminogluc6sido, la netilmicina, de reciente introducción. Se presentan los porcentajes de inhibición logrados por los antibióticos probados, para cada microorganismo, ordenados en magnitudes decrecientes. Se recomienda este tipo de estudios para tener un conocimiento básico relativo al comportamiento de las cepas bacterianas de una comunidad frente a los antibióticos.
\end{abstract}

Con el advenimiento de las sulfas y de la penicilina por los años cuarenta de este siglo, se inició para el sector salud la era de los antibióticos, como elementos de primera línea en el tratamiento de las enfermedades infecciosas. De entonces acá, son muchas y muy variadas las sustancias antibióticas que se han puesto al servicio de la humanidad y ha sido muy patente la universalización de su empleo.

El hecho de que el mundo de la ciencia se haya y se halle empeñado en la consecución de nuevas sustancias de esta índole se debe a la necesidad sentida de lograrlas con un amplio espectro antimicrobiano, a las reacciones adversas ocurridas en algunas de las personas que las reciban y a la resistencia natural o adquirida que pueden los microorganismos, o cepas de ellos, o tales o cuales antibióticos.

La conducta ideal a seguir frente a todo proceso de infección, antes de instaurar un tratamiento con antibióticos, es el aislamiento, identificación y tipificación del germen causal, seguidos de la prueba in vitro para medir la sensibilidad de éste a las sustancias antibióticas. Sin embargo, hay circunstancias por las cuales no se puede cumplir este postulado; por ejemplo, cuando la gravedad del cuadro clínico obliga una atención inmediata o cuando se carece de parte o de todos los elementos necesarios para cumplir el proceso, caso no poco infrecuente en nuestro medio.

* Profesor Asociado, Facultad de Medicina, U.N.

** Jefe Sección Diagnóstico, Investigación y Referencia, Instituto Nacional (INS). Profesor Asociado, Facultad de Medicina, U.N.

*** Bacterióloga Unidad de Enterobacterias. Grupo de Microbiología e Inmunología INS.

**** Coordinación Epidemiológica INS. 
Conviene entonces tener un conocimiento epidemiológico de base, sobre el comportamiento de los microorganismos más comunes en una región, frente a los antibióticos disponibles en ella. El trabajo que ahora se presenta se entiende como un primer aporte al cumplimiento del objetivo esbozado, probando específicamente la netilmicina, amino-glucósido de reciente introducción en nuestro medio.

\section{MATERIALES Y METODOS}

\section{Características del Trabajo}

Por razones del tamaño de las muestras, que para ninguna de las cepas estudiadas sobrepasó la cantidad de cincuenta, y por tenerse como norma el establecer un punto límite fijo para determinar la ocurrencia o no ocurrencia (p.q.) del fénómeno de sensibilidad de las cepas a los antibióticos, este estudio tiene un cariz apenas descriptivo, por cuanto no caben, salvo casos extremos, las pruebas de significación estadística para las diferencias entre las resultantes. Los cálculos de medidas de tendencia central que se hicieron sobre los diámetros de los halos de inhibición se presentan a manera especulativa, con miras a que el observador pueda tener idea de los posibles hallazgos cuando aplique un antibiograma a determinado germen.

Cepas : Las cepas del presente estudio fueron aisladas de casos clínicos (no discriminados según condición alguna), en diferentes centros asistenciales de Colombia, referidas al INS para confirmación. Toda cepa fue estudiada exhaustivamente desde el punto de vista bioquímico y serotipificada cuando fue necesario. Solamente fueron aceptables para el estudio las cepas completamente indentificadas. En total se seleccionaron 302 cepas.

Medios : Para los estudios bacteriológicos se utilizaron los medios convencionales recomendados por el CDC. Para conservación de cepas se utilizaron tubos de agar nutritivo inclinado. Para las pruebas de sensibilidad se utilizó como medio, MullerHinton (BBL 11438). Los discos de antibió- ticos se obtuvieron comercialmente (BBL. P.o. Box 243 Cockesville, MD 21030 USA).

Siguiendo la técnica de Kirby Bauer (1) recomendada por el CDC (2), cada cepa en estudio fue cultivada en forma pura sobre agar nutritivo; luego se seleccionaron 4 o 5 colonias las cuales fueron resembradas en tubos que contenían $5 \mathrm{ml}$. de caldo de Muller-Hinton. Después de un período de incubación de 4 horas se ajustó la turbidez de crecimiento al tubo 0,5 de la escala de Mc-Farland.

Este cultivo se sembró utilizando aplicadores de algodón, con cubrimiento de la superficie tersa de una caja de MullerHinton. Sobre esta siembra se colocaron los discos con los respectivos antibióticos. Para cada grupo de pruebas realizadas se practicó un control consistente en el antibiograma para las cepas de sensibilidad conocida S. aureus (ATCC-25923), E. coli (ATCC25922), y $P$. aeruginosa (ATCC-27853). Después de la incubación de 18 horas a $37^{\circ} \mathrm{C}$ los halos de inhibición fueron leídos con un lector semiautomático de pantalla. Los datos fueron cuidadosamente registrados para su evaluación posterior.

Para efectos de estimar el grado de inhibición en el crecimiento y desarrollo bacterianos se consideraron los diámetros de los halos de inhibición, tomando como valores de sensibilidad los de 12 o más milímetros para las Pseudomonas y los de 17 o más milímetros para el resto de los microorganismos estudiados (1-2).

El número de cepas estudiadas por cada microorganismo no fue constante por el hecho, entre otros, de la poca frecuencia con que se encuentran algunos de ellos como agentes etiológicos de los fenómenos infecciosos; hubo así gérmenes de los que se estudia ron sólo tres o cuatro cepas, lo cual hizo que para el análisis estadístico se les agrupara por géneros (Cuadro No. 1).

\section{Antibióticos :}

Los sensidiscos que se emplearon para las pruebas, impregnados con el antibiótico, tenían las siguientes concentraciones del producto: 
Amikacina

Ampicilina

Cefalotina

Cloranfenicol

Gentamicina

Kanamicina

Tetraciclina

Netilmicina

Sisomicina

Trimethoprim-sulfa

CUADRO № I. Cepas bacterianas sometidas a antibiograma, según: género, especie, serotipo o agrupación.

\begin{tabular}{|c|c|c|c|}
\hline I. & Escherichia coli & & 50 \\
\hline 2. & Salmonella typhi & & 50 \\
\hline 3. & Salmonella enteritidis ser. Agona & 18 & \\
\hline 4. & Salmonella enteritidis ser. Typhimurium & 4 & \\
\hline 5. & Salmonella enteritidis ser. Manhattan & 4 & \\
\hline 6. & Salmonella enteritidis ser. Dublin & 4 & \\
\hline 7. & Salmonella enteritidis ser. Gallinarum & 3 & \\
\hline & TOTAL Salmonella enteritidis & & 33 \\
\hline 8. & Shigella flexneri & 10 & \\
\hline 9. & Citrobacter freundii & 10 & \\
\hline 10. & Proteus mirabilis & 10 & \\
\hline 11. & Morganella morganii & 10 & \\
\hline 12. & Klebsiella pneumoniae & 10 & \\
\hline 13. & Serratia liquefaciens & 5 & \\
\hline 14. & Yersinia enterocolítica & 4 & \\
\hline & TOTAL diferentes enterobacteriaceae & & 59 \\
\hline 15. & Staphylococcus aureus coagulasa positiva & & 28 \\
\hline 16. & Pseudomonas s.p. & & 50 \\
\hline 17. & Gérmenes no fermentadores & & 32 \\
\hline & TOTAL & & 302 \\
\hline
\end{tabular}

\section{RESULTADOS}

En el caso de Escherichia coli, ninguno de los antibióticos alcanzó la inhibición para el $100 \%$ de las cepas (Cuadro No. 2 y Gráfica No. 1). Los antibióticos con los cuales se obtuvieron los mejores resultados fueron en su orden, Amikacina (96\% de inhibición), Trimethoprim-sulfa $(94 \%)$ y Netilmicina $(90 \%)$. El promedio calculado para los diámetros del total de los halos de inhibición superiores a $17 \mathrm{~mm}$. para todos los antibióticos probados, fue de 21. 17, con una desviación standard de 4,0 .

De las 50 cepas de E. coli probadas, una fue resistente a nueve antibióticos (salvo Amikacina); dos lo fueron a 7 antibióticos; una resistente a 6 ; una resistente a 5 ; trece cepas resistentes a 4 antibióticos; doce resistentes a 3 antibióticos: diez resistentes a 2 antibióticos, de las cuales, seis fueron a Ampicilina, Cephalotina; y diez cepas resistentes a un solo antibiótico. siendo 9 de ellas a Ampicilina.

La más alta frecuencia de sensibilidad de las cepas de Salmonella typhi se obtuvo con Cephalotina y Amikacina, donde se cubrió el

CUADRO № 2. Distribución Porcentual de las cepas de Escherichia colt estudiadas, por grupos de diámetros de los halos de Inhibición (en $\mathrm{mm}$ ), según antibióticos.

\begin{tabular}{|c|c|c|c|c|c|c|c|}
\hline \multirow{2}{*}{ ANTIBIOTICO } & \multicolumn{7}{|c|}{ DIAMETRO DEL HALO DE INHIBICION EN $\mathrm{mm}$} \\
\hline & $\leq 6$ & $7-11$ & $12-16$ & $17-21$ & $22-26$ & $27-31$ & $\geq 32$ \\
\hline Amikacina & - & 2 & 2 & 58 & 38 & - & - \\
\hline Trimethoprim-sulfa & - & 6 & - & 32 & 42 & 20 & - \\
\hline Netilmicina & - & - & 10 & 52 & 38 & - & - \\
\hline Sisomicina & 4 & - & 10 & 66 & 20 & - & - \\
\hline Gentamicina & 2 & - & 12 & 60 & 22 & 4 & - \\
\hline Kanamicina & 14 & 2 & 10 & 52 & 22 & - & - \\
\hline Cloranfenicol & 24 & - & 14 & 10 & 28 & 24 & - \\
\hline Tetraciclina & 32 & 8 & 4 & 46 & 10 & - & - \\
\hline Cephalotina & 16 & 12 & 28 & 38 & 6 & - & - \\
\hline Ampicilina & 54 & 40 & 4 & 2 & - & - & - \\
\hline
\end{tabular}

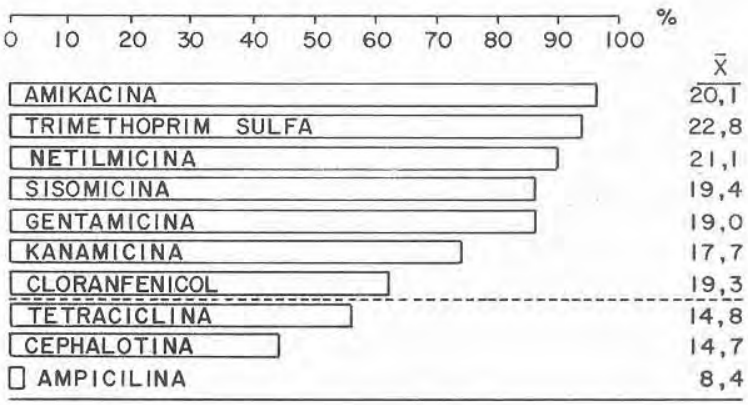

GRAFICA. № I. Porcentajes de las cepas de E. coli inhibidas por los antibióticos probados. Promedios de los diámetros de los halos de inhibición. 
$100 \%$ de inhibición del crecimiento, con halos de diámetro de 17 o más mm (Cuadro No. 3 y Gráfica No. 2); en orden de frecuencia decreciente siguieron, Trimethoprim-sulfa, Tetraciclina y Kanamicina, con un $98 \%$ de inhibición cada una; luego vinieron: Cloranfenicol (94\%), Netilmicina y Sisomicina (92\% c/u.), Gentamicina $(86 \%)$ y Ampicilina (74\%).

El promedio en los halos de 17 o más $\mathrm{mm}$ de diámetro, para todos los antibíbticos fue de 23,57 con una desviación standard de 5,2.

Veintitres de las 50 cepas de S. typhi estudiadas presentaron resistencia a alguno de los antibióticos probados. Diez y siete presentaron resistencia a solo un antibiótico, siendo el más común (9) Ampicilina; una cepa presentó resistencia a dos antibióticos. Trece de las 23 cepas que presentaron resistencia, lo fueron a la Ampicilina.

CUADRO № 3. Distribución Porcentual de las cepas de Salmonella typhi estudiadas, por grupos de diámetros de los halos de inhibición (en mm), según antibióticos.

\begin{tabular}{|c|c|c|c|c|c|c|c|}
\hline \multirow{2}{*}{ ANTIBIOTICOS } & \multicolumn{2}{|c|}{ DIAMETRO } & \multicolumn{5}{|c|}{ DEL HALO DE INHIBICION EN mm } \\
\hline & $\leq 6$ & $7-11$ & $12-16$ & $17-2 \mid$ & $22-26$ & $27-31$ & $\geq 32$ \\
\hline Cephalot ina & - & - & - & - & 32 & 60 & 8 \\
\hline Amikacina & - & - & - & 24 & 70 & 6 & - \\
\hline Trimethopr im-sulfa & - & 2 & - & 4 & 12 & 42 & 40 \\
\hline Tetraciclina & - & - & 2 & 6 & 50 & 38 & 4 \\
\hline Kanamicina & - & 2 & - & 16 & 70 & 12 & - \\
\hline Cloranfenicol & - & 2 & 4 & 50 & 42 & 2 & - \\
\hline Netilmicina & - & - & 8 & 32 & 44 & 16 & - \\
\hline Sisomicina & - & - & 8 & 42 & 50 & - & - \\
\hline Gentam icina & - & - & 16 & 32 & 44 & 6 & 2 \\
\hline Ampicilina & - & 2 & 24 & 60 & 14 & - & - \\
\hline
\end{tabular}

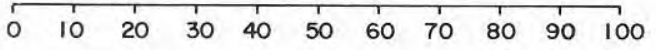

\begin{tabular}{|c|c|}
\hline CEPHALOTINA & $\overline{28,4}$ \\
\hline AMIKACINA & 22,9 \\
\hline TRIME THOPRIM SULFA & 29,9 \\
\hline IETRACICLINA & 26,2 \\
\hline KANAMICINA & 23,8 \\
\hline CLORANFENICOL & 21,3 \\
\hline NET ILMICINA & 22,8 \\
\hline SISOMICINA & 21,5 \\
\hline GENTAMICINA & 21,5 \\
\hline AMPICILINA & 18,8 \\
\hline
\end{tabular}

GRAFICA № 2. Porcentajes de cepas de Saimonella typhi inhibidas por los antibióticos probados. Prome dios de los diámetros de los halos de inhibición.
El comportamiento de los antibióticos probados frente a Salmonella enteritidis serotipo Agona fue muy dispar, con diferencias francamente notorias y significativas. Siete de ellos no alcanzaron la inhibición de siquiera el $50 \%$ de las cepas, siendo el promedio de los diámetros de los halos de inhibición, menor de $9 \mathrm{~mm}$ en cuatro de estos: Kanamicina, Ampicilina, Tetraciclina y Amikacina alcanzaron el $100 \%$ de inhibición de $17 \mathrm{~mm}$ o más. El promedio de los diámetros de los halos de inhibición, para todos los antibióticos probados, iguales o superiores $17 \mathrm{~mm}$, fue de $23,16 \mathrm{~mm}$, con desviación estandar de 2,9.

De las 18 cepas de S. enteritidis serotipo Agona solamente 3 fueron inhibidas por los diez antibióticos probados. La resistencia de las 15 restantes fue de una para cuatro antibióticos, una para cinco, una para seis y de 12 para siete.

CUADRO № 4. Distribución porcentual de las cepas de Salmonella enteritidis serotipo agona estudiadas, por grupos de diámetros de los halos de inhibición (en $\mathrm{mm}$ ), según antibióticos.

\begin{tabular}{|l|c|c|c|c|c|c|c|}
\hline \multirow{2}{*}{ ANTIBIOTICOS } & \multicolumn{8}{|c|}{ DIAMETRO } & DEL HALO DE & INHIBICION EN mm \\
\cline { 2 - 8 } & $\leq 6$ & $7-11$ & $12-16$ & $17-21$ & $22-26$ & $27-31$ & $\geq 32$ \\
\hline Trimethoprim-sulfa & - & - & - & 5,6 & 72,2 & 22,2 & - \\
Netilmicina & - & - & - & 50,0 & 50,0 & - & - \\
Amikacina & - & - & - & 77,8 & 22,2 & - & - \\
Sisomicina & 5,6 & 33,3 & 27,8 & 11,1 & 22,2 & - & - \\
Cephalotina & - & 27,8 & 44,4 & 16,7 & - & 11,1 & - \\
Gentamicina & 44,4 & 27,8 & 5,6 & 11,0 & 5,6 & - & 5,6 \\
Kanamicina & 72,2 & 11,1 & - & 16,7 & - & - & - \\
Ampicilina & 83,3 & - & - & 16,7 & - & - & - \\
Tetraciclina & 77,8 & - & 11,1 & 11,1 & - & - & - \\
Cloranfenicol & 77,8 & 5,6 & 5,6 & 11,0 & - & - & - \\
\hline
\end{tabular}

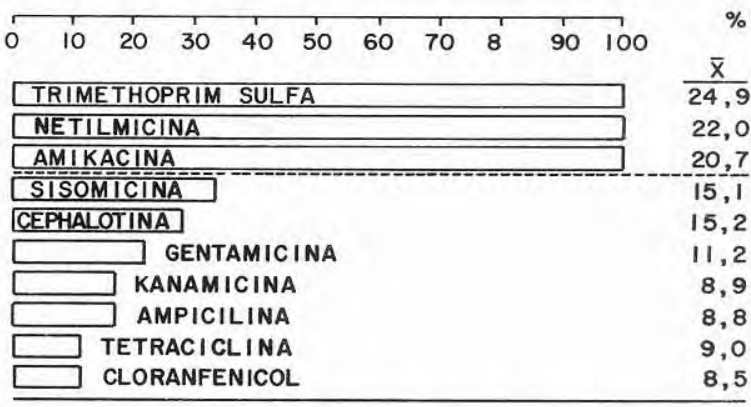

GRAFICA N2 3. Porcentajes de cepas de Salmonella enteritidis serotipo ggong inhibidas por los antibióticos probados. Promedios de los diámetros de los halos de inhibición. 
Como se indicó antes, debido a que el número de cepas logradas de los otros cuatro serotipos de $S$ enteritidis no fue suficiente para hacer su análisis individual, se optó por tratarlas en conjunto, añadidas las del serotipo Agona, teniendo en cuenta que para la gran mayoría de los laboratorios no cabe la posibilidad de hacer serotipificaciones y por tanto esta especie de germen es tomada solamente como tal. Las 33 cepas de Salmonella enteritidis estudiadas fueron inhibidas en su totalidad $(100 \%)$ por Netilmicina (Cuadro No. 5 y Gráfica No. 4); Amikacina inhibió el 97\% y Trimethoprimsulfa el 93,9\%; el porcentaje de inhibición de los demás antibióticos no amerita que se les tome en cuenta. El promedio de los diámetros de inhibición de $17 \mathrm{~mm}$ o más, fue de 22,10 , $\mathrm{mm}$, con una desviación estandar de 3,4 . Son muy claros en el Cuadro No. 5 los altos porcentajes de cepas de diámetros de inhibición de $6,99 \mathrm{~mm}$ o menos hallados para cuatro antibióticos que suelen tomarse como elementos de primera línea en el tratamiento de diarrea y enteritis: Kanamicina $(42,4 \%)$ Cloranfenicol $(48,5 \%)$ Tetraciclina $(54,5 \%)$ y Ampicilina $(63,6 \%)$.

Apenas cuatro de las 33 cepas que se estudiaron en el grupo de Salmonella enteritidis fueron inhibidas por los 10 antibióticos en prueba. Tres cepas fueron resistentes a un solo antibiótico; siete lo fueron a dos antibióticos; dos, a tres y cuatro antibióticos, respectivamente; una a cinco y una a seis antibióticos y trece a siete de los 10 antibióticos probados. Conviene destacar para este grupo, que el $39,4 \%$ de las cepas

CUADRO № 5. Distribución porcentual de las cepas de Salmonella enteritidis estudiadas, por grupos de diámetros de los halos de inhibi ción (en $\mathrm{mm})$, según antibióticos.

Serotipo ggona (I8 cepas), dublin (4), typhimurium (4), manhattan (4) y gallinarum (3).

\begin{tabular}{|l|r|r|r|r|r|r|r|}
\hline \multirow{2}{*}{ ANTIBIOTICOS } & \multicolumn{3}{|c|}{ DIAMETRO } & DEL HALO & DE & \multicolumn{1}{|c|}{ HIBICION EN mm } \\
\cline { 2 - 8 } & $\leq 6$ & $7-\mid 1$ & $12-16$ & $17-21$ & $22-26$ & $27-31$ & $\geq 32$ \\
\hline Netilmicina & - & - & - & 33,3 & 66,7 & - & - \\
Amikacina & - & - & 3,0 & 78,8 & 18,2 & - & - \\
Trimethoprim-sulfa & 6,1 & - & - & 3,0 & 66,7 & 24,2 & - \\
Sisomicina & 3,0 & 21,2 & 15,2 & 15,2 & 42,4 & 3,0 & - \\
Cephalotina & 9,1 & 24,2 & 24,2 & 9,1 & 18,2 & 15,2 & - \\
Gentamicina & 27,3 & 15,2 & 6,0 & 33,3 & 15,2 & - & 3,0 \\
Kanamicina & 42,4 & 6,1 & 3,0 & 39,4 & 9,0 & - & - \\
Tetraciclina & 54,5 & - & 9,1 & 27,3 & 6,1 & 3,0 & - \\
Cloranfenicol & 48,5 & 3,0 & 21,2 & 21,2 & 6,1 & - & - \\
Ampicilina & 63,6 & 9,1 & 6,1 & 15,1 & 6,1 & - & - \\
\hline
\end{tabular}

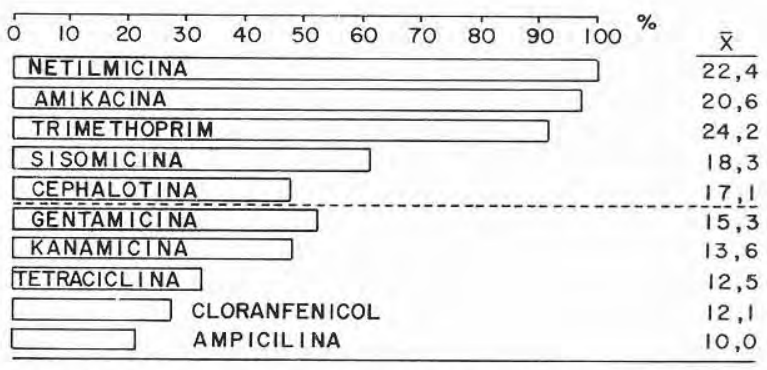

GRAFICA № 4. Porcentajes de cepas de Salmonellas enteritidis estudiadas inhibidas por los antibióticos probados. Promedios de los diámetros de los halos de inhibición.

Serotipo ggona (18), dublin (4), fyphimurium (4), manhattan (4), gallinarum (3).

presentaron resistencia a 7 antibióticos y solo el $12,1 \%$ fue sensible a alguno de los antibióticos probados.

El grupo de diferentes miembros de la familia Enterobacteriacea quedó conformado para el análisis, por las siguientes especies: Shigella flexnerii, Citrobacter freundii, Proteus mirabilis, Morganella morganii y Klebsiella pneumoniae con 10 cepas cada una, Serratia liquefaciens con 5 cepas y Yersinia enterocolítica con cuatro.

El único antibiótico que alcanzó el 100\% de inhibición, con halos de 17 o más mm de diámetro, fue Sisomicina (Gráfica No. 5 y Cuadro No. 6). Trimethoprim-sulfa y Netilmicina inhibieron el $98,3 \%$ cada uno. Gentamicina es también efectiva, con el $96,6 \%$ de cepas inhibidas. El promedio de los

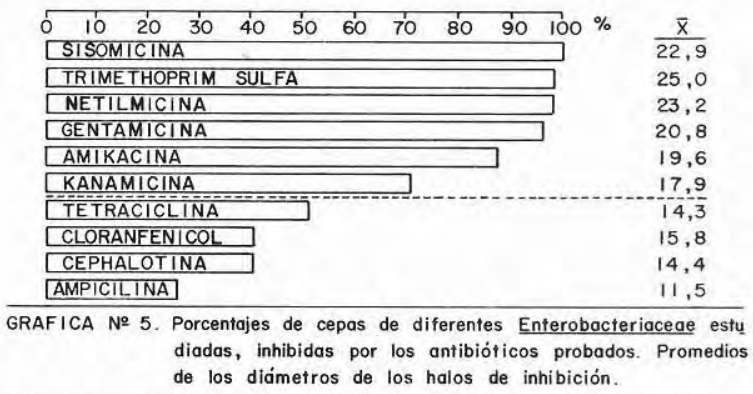

Shigella flexnerii (10 cepas), Citrobacter freundii (10), Proteus mirábilis (10), Morganella morganii (10), Klebsiella pneumoniae (10), Serratia liquefaciens (5) y Yersinia enterocolitica (4) 
CUADRO № 6. Distribucion porcentual de las cepas de diferentes Enterobacteriaceae estudiadas, por grupos de los diámetros de inhibición (en $\mathrm{mm}$ ), según antibióticos.

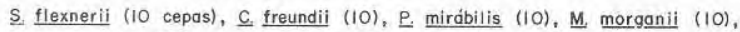
$K$. pneumoniae $(10), S$. liguefaciens (5), y $\underline{Y}$ enterocolítica (4)

\begin{tabular}{|l|c|c|c|ccc|c|}
\hline \multirow{2}{*}{ ANTIBIOTICOS } & \multicolumn{3}{|c|}{ DIAMETRO DEL HALO DE } & \multicolumn{3}{c|}{ INHIBICION EN mm } \\
\cline { 2 - 8 } & $\leq 6$ & $7-11$ & $12-16$ & $17-21$ & $22-26$ & $27-31$ & $\geq 32$ \\
\hline Sisomicina & - & - & - & 40,6 & 54,1 & 5,3 & - \\
Trimethoprim-sulfa & - & 1,7 & - & 28,8 & 27,1 & 42,4 & - \\
Netilmicina & - & - & 1,7 & 32,2 & 55,9 & 10,2 & - \\
Gentamicina & - & - & 3,4 & 64,4 & 32,2 & - & - \\
Amikacina & 1,7 & - & 10,2 & 71,2 & 16,9 & - & - \\
Kanamicina & 11,9 & - & 16,9 & 55,9 & 15,3 & - & - \\
Tetraciclina & 30,5 & 8,5 & 10,2 & 44,0 & 3,4 & 3,4 & - \\
Cloranfenicol & 11,8 & 18,6 & 28,8 & 27,1 & 8,4 & 5,3 & - \\
Cephalotina & 30,5 & 10,2 & 18,6 & 23,7 & 15,3 & 1,7 & - \\
Ampicilina & 37,3 & 28,7 & 8,4 & 20,3 & 5,3 & - & - \\
\hline
\end{tabular}

diámetros de los halos de inhibición de 17 mm o superiores fue de 21,73 y la desviación estandar de 3,74 .

En el Cuadro No. 7 se ve claramente cómo las cepas de las especies de Enterobacteriacea presentaron resistencia, al menos a uno de los antibióticos probados. En la mayoría de las especies, excepto Shigella flexnerii las cepas resistentes lo fueron a tres o más antibióticos; el 62.7\% de las cepas estuvieron en tal circunstancia.

Para las cepas de Staphylococcus aureus coagulasa positiva, se observó que, cuatro de los antibióticos probados inhibieron el crecimiento y desarrollo del total de las cepas; ellos en su orden decreciente, según el diámetro de los halos alcanzados (Gráfica No. 6 y Cuadro No. 8), fueron: Cephalotina, Trimethoprim-sulfa, Kanamicina y Netilmi-

CUADRO № 7. Distribución de las cepas de las diferentes Enterobacteriaceae estudiadas, por número de antibióticos a que fueron resistentes y según especie bacteriana.

\begin{tabular}{|c|c|c|c|c|c|c|c|c|}
\hline \multirow{2}{*}{$\begin{array}{c}\text { ESPECIES } \\
\text { BACTERIANAS }\end{array}$} & \multirow{2}{*}{$\begin{array}{c}\text { CEPAS } \\
\text { SIN } \\
\text { RESIST. }\end{array}$} & \multicolumn{6}{|c|}{ CEPAS RESISTENTES } & \multirow{2}{*}{ TOTAL } \\
\hline & & 1 & 2 & 3 & 4 & 5 & 6 & \\
\hline Shigella flexneri & - & 4 & 6 & - & - & - & - & 10 \\
\hline Citrobacter freundii & - & 3 & 2 & 5 & - & - & - & 10 \\
\hline Proteus mirabilis & - & - & 2 & 4 & 2 & 2 & - & 10 \\
\hline Morganelia morganit & - & - & - & 4 & 4 & 1 & 1 & 10 \\
\hline Klebsiella pneumoniae & - & 1 & 1 & 4 & 3 & 1 & - & 10 \\
\hline Serratia liquefaciens & - & - & - & 2 & 2 & 1 & - & 5 \\
\hline Yersinia enterocolitica & - & 1 & 2 & 1 & - & - & - & 4 \\
\hline TOTAL & - & 9 & 13 & 20 & 11 & 5 & 1 & 59 \\
\hline
\end{tabular}

cina. En los demás antibióticos hubo resistencia presentando las menores proporciones Gentamicina $(3,6 \%)$ y Sisomicina $(7.2 \%)$; el antibiótico menos operante fue Ampicilina, con el 96,4\% de resistencia. El promedio de los diámetros de los halos de inhibición de 17 o más mm. fue de 23.0 y su desviación estandar de 4,8.

Todas las cepas de Staphylococcus aureus coagulasa positiva que se estudiaron fueron resistentes a algún antibiótico; seis cepas lo fueron a uno de los antibióticos, 16 a dos de ellos, 4 a tres y una a cuatro y a cinco, respectivamente. Así, pues, el $21,4 \%$ de las cepas fueron resistentes a 3 o más antibióticos.

Frente a las Pseudomonas sp. el comportamiento de los antibióticos probados fue deficiente para la inhibición del crecimiento

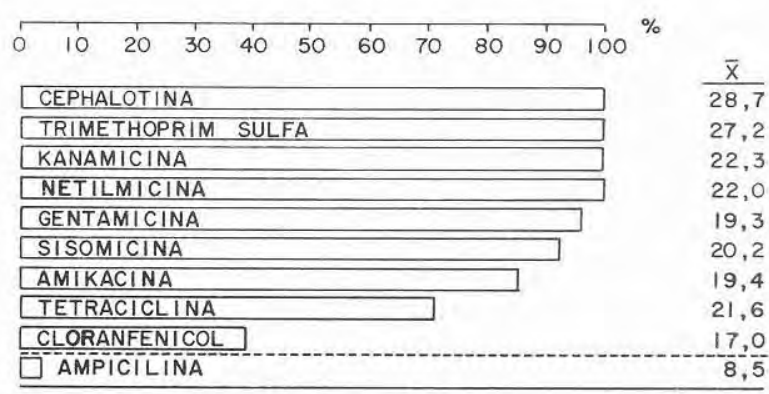

GRAFICA № 6. Porcentajes de cepas de Staphylococcus aureus coagulasa positiva inhibidas por los antibióticos probados. Promedios de los diámetros de los halos de inhibición, en $\mathrm{mm}$.
CUADRO № 8. Distribución porcentual de las cepas de Staphylococcus aureus coagulasa positiva estudiadas, pur grupos de los diámetros de los halos de inhibición (en $\mathrm{mm}$ ), según antibióticos em pleados.

\begin{tabular}{|l|c|c|c|c|c|c|c|}
\hline \multirow{2}{*}{ ANTIBIOTICOS } & \multicolumn{8}{|c|}{ DIAMETRO DEL HALO DE INHIBICION EN $\mathrm{mm}$} \\
\cline { 2 - 8 } & $\leq 6$ & $7-11$ & $12-16$ & $17-2 \mid$ & $22-26$ & $27-31$ & $\geq 32$ \\
\hline Cephalotina & - & - & - & 3,6 & 10,7 & 82,1 & 3,6 \\
Trimethoprim-sulfo & - & - & - & 7,1 & 42,9 & 39,3 & 10,7 \\
Kanamicina & - & - & - & 53,6 & 35,7 & 10,7 & - \\
Netilmicina & - & - & - & 53,6 & 42,9 & 3,5 & - \\
Gentamicina & 3,6 & - & - & 85,7 & 10,7 & - & - \\
Sisomicina & 3,6 & - & 3,6 & 75,0 & 17,8 & - & - \\
Amikacina & - & 10,7 & 3,6 & 67,9 & 17,8 & - & - \\
Tetraciclina & - & 10,7 & 17,9 & 7,1 & 50,0 & 14,3 & - \\
Cloranfenicol & 7,2 & 3,6 & 50,0 & 17,8 & 17,8 & 3,6 & - \\
Ampicilina & 28,5 & 67,9 & - & - & 3,6 & - & - \\
\hline
\end{tabular}


y desarrollo de las cepas, (Gráfica No. 7 y Cuadro No. 9). Amikacina, el mejor de ellos, inhibió el $94 \%$ de las cepas. Le siguió en importancia Netilmicina, con el $84 \%$ de cepas inhibidas. El promedio de los diámetros de los halos de inhibición de $12 \mathrm{~mm}$ o mayores, fue de 17,88 . Observando la dispersión de los diámetros de los halos, se obtiene la demostración de que las diferentes cepas de Pseudomonas sp. tienen tendencia a ser, o francamente resistentes a un antibiótico o marcadamente sensibles a él.

Ninguna de las 50 cepas de Pseudomonas sp. que se estudiaron dejó de presentar resistencia al menos a un antibiótico. Apenas el 2\% fueron resistentes a un solo antibiótico; el $4 \%$ a 2 antibióticos; el $12 \%$ a 3 antibióticos; el $4 \%$ a 4 antibióticos; el $10 \%$ a 5 antibióticos; el $34 \%$ a 6 antibióticos; el 6\% a 9 antibióticos y el $4 \%$ a los 10 antibióticos probados. El hecho de que el $44 \%$ de las

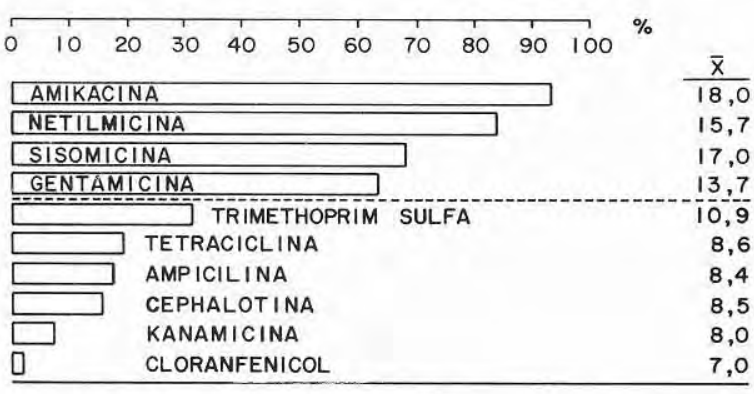

GRAFICA № 7. Porcentajes de cepas de Pseudomonas $\underline{s} p$. inhibidas por los antibióticos probados. Promedios de los diámetros de los halos de inhibición, en $\mathrm{mm}$.

CUADRO № 9. Distribución porcentual de las cepas de Pseudomonas s $p$ estudiadas, por grupos de los diámetros de los halos de inhibición (en $\mathrm{mm}$ ), según antibióticos probados.

\begin{tabular}{|c|c|c|c|c|c|c|c|}
\hline \multirow{2}{*}{ ANTIBIOTICOS } & \multicolumn{2}{|c|}{ DIAMETRO } & \multicolumn{2}{|c|}{ DEL HALO DE } & \multicolumn{3}{|c|}{ INHIBICION EN mm } \\
\hline & $\leq 6$ & $7-11$ & $12-16$ & $\mid 7-21$ & $22-26$ & $27-31$ & $\geq 32$ \\
\hline Amikacina & - & 6,0 & 30,0 & 54,0 & 10,0 & - & - \\
\hline Netilmicina & 14,0 & 2,0 & 40,0 & 32,0 & 12,0 & - & - \\
\hline Sisomicina & 18,0 & 14,0 & 8,0 & 20,0 & 38,0 & 2,0 & - \\
\hline Gentamicina & 24,0 & 12,0 & 32,0 & 30,0 & 2,0 & - & - \\
\hline Trimethoprim-sulfa & 68,0 & - & 10,0 & 10,0 & 10,0 & 2,0 & - \\
\hline Tetraciclina & 66,0 & 14,0 & 16,0 & 4,0 & - & - & - \\
\hline Ampicilina & 82,0 & - & 8,0 & 8,0 & 2,0 & - & - \\
\hline Cloranfenicol & 78,0 & 20,0 & - & 2,0 & - & - & - \\
\hline
\end{tabular}

cepas haya sido resistente a 6 o más antibióticos demuestra muy enfáticamente el problema que este microorganismo plantea en clínica.

El grupo de microorganismos no fermen tadores tuvo también un marcado grado de resistencia a los antibióticos contra los cuales se les probó (Gráfica No. 8 y Cuadro No. 10). Amikacina y Sisomicina apenas alcanzaron respectivamente el $65,6 \%$ de inhibición de cepas. El promedio de los diámetros de los halos de inhibición, de 17 $\mathrm{mm}$ o mayores fue relativamente alto $(19,21)$ con una desviación estandar de 3,8. En estos gérmenes, como en las Pseudomonas sp. se observa el fenómeno de que las cepas resistentes tienden a serlo francamente.

Del total de 32 cepas de microorganismos no fermentadores probados. todas fueron resistentes a tres o más antibióticos; el $75 \%$

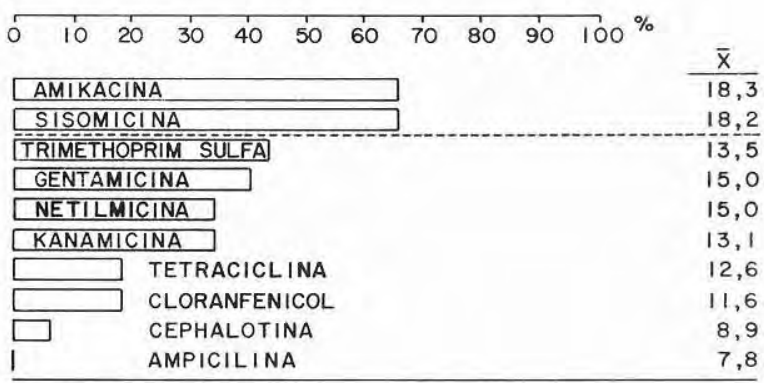

GRAFICA № 8. Porcentajes de cepas de Gérmenes no fermentadores inhibidas por los antibióticos probados. Promedios de los diámetros de los halos de inhibición.
CUADRO № 10. Distribución porcentual de las cepas de Gérmenes no fermentadores estudiadas, por grupos de diámetros de los halos de inhibición (en $\mathrm{mm}$ ), según antibióticos probados.

\begin{tabular}{|l|rrr|r|r|r|r|r|}
\hline \multirow{2}{*}{ ANTIBIOTICOS } & \multicolumn{6}{|c|}{ DIAMETRO DEL HALO DE INHIBICION EN $\mathrm{mm}$} \\
\cline { 2 - 8 } & $\leq 6$ & $7-11$ & $12-16$ & $17-21$ & $22-26$ & $27-31$ & $\geq 32$ \\
\hline Amikacina & - & - & 34,4 & 62,5 & 3,1 & - & - \\
Sisomicina & 6,3 & - & 28,1 & 50,0 & 15,6 & - & - \\
Trimethoprim-sulfa & 34,4 & 12,5 & 9,4 & 28,1 & 15,6 & - & - \\
Gentamicina & 12,5 & 9,4 & 37,5 & 34,4 & 6,2 & - & - \\
Netilmicina & 12,5 & 15,6 & 37,5 & 34,4 & - & - & - \\
Kanamicina & 37,5 & 6,2 & 21,9 & 31,3 & - & 3, & - \\
Tetraciclina & 25,0 & 15,6 & 40,6 & 18,8 & - & - & - \\
Cloranfenicol & 31,2 & 28,1 & 21,9 & 18,8 & - & - & - \\
Ampicilina & 59,4 & 40,6 & - & - & - & - & - \\
\hline
\end{tabular}


de ellas lo fueron a seis o más antibióticos. El porcentaje acumulativo en las resistencias de cepas, según orden descendente en el número de antibióticos, fue como sigue: resistentes a 10 antibióticos $9,4 \%$; a 9 o más, $21,9 \%$; a 8 o más, $31,3 \%$; a 7 o más, $50.0 \%$; a 6 o más, $75,0 \%$ a 3 o más, $87,5 \%$; a 40 más, $90,6 \%$ a 3 o más $100,0 \%$.

\section{DISCUSION}

La Netilmicina (Sulfato de Netilmicina), es un aminoglucósido semisintético, cuya especial cualidad frente a otros aminoglucósidos radica en que no es inactivado por las enzimas adenililantes (3) propias de varios microorganismos, principalmente de la familia Enterobacteriácea; por otra parte es menos ototóxica y nefrotóxica, como se demostró experimentalmente (4).

Descontado el objetivo de análisis de la Netilmicina, este tipo de estudios se considera de gran valor para la orientación de quienes tienen que ordenar o practicar antibiogramas para determinar la sensibilidad de los microorganismos patógenos aislados y de quienes, sin poder contar con el auxilio total o parcial de un laboratorio bacteriológico, deben adoptar conductas terapéuticas a la luz simplemente de la clínica.

En infecciones por Escherichia coli los antibióticos de elección para los antibiogramas o para la terapéutica estarían, en su orden de beneficio, constituidos por Amikacina, Trimethoprim-sulfa y Netilmicina.

Para Salmonella typhi, habida cuenta de la aceptación universal del cloranfenicol, se encuentran Cephalotina y Amikacina como los antibióticos de mejor opción y Ampicilina como el que merece no tomarse en cuenta. Tetraciclina, Netilmicina y Sisomicina estarían en segunda opción.

En Salmonella enteritidis serotipo Agona, prácticamente cabe solo pensar en los tres antibióticos (Trimethoprim-sulfa, Netilmicina y Amikacina) que alcanzaron un cubri- miento del $100 \%$ en su acción. La opción de los demás estudiados es bastante pobre. Importa recalcar en este hallazgo, frente al amplio uso que suele hacerse de varios de ellos para tratar problemas de diarreas y enteritis. Al tomar en conjunto las Salmonellas enteritidis queda Netilmicina como el antibiótico de elección, y Amikacina y Trimethoprim-sulfa como segunda y tercera opciones.

El antibiótico de primera línea para los casos de infección por otros miembros de la familia Enterobacteriácea es Sisomicina, seguido en estrecha importancia por Trimethoprim-sulfa, Netilmicina y Gentamicina; como descartables están la Tetraciclina, el Cloranfenicol, la Cephalotina y la Ampicilina.

Tomando en conjunto los microorganismos entéricos estudiados (excluída Salmonella typhi por su calidad etiopatogénica específica) se tiene que el antibiótico mejor es Netilmicina (98,9\% de sensibilidad); le sigue Trimethoprim-sulfa $(96,7 \%)$ y Amifacina $(91,3 \%)$. Los demás antibióticos no alcanzaron el $90 \%$ y como ya se mencionó, Ampicilina, Cephalotina, Cloranfenicol, Kanamicina y Tetraciclina, tuvieron resultantes inconsistentes.

Para Staphylococcus aureus coagulasa positiva, en el presente trabajo aparecen cuatro antibióticos útiles: Cephalotina, Trimethoprim-sulfa, Kanamicina y Netilmicina. Gentamicina y Sisomicina entran a la segunda línea de opciones. Los demás antibióticos no alcanzaron a inhibir el 90\% de las cepas.

Referente a las Pseudomonas sp., las posibilidades están limitadas. El antibiótico de elección sería Amikacina; en segunda instancia Netilmicina y en tercer Sisomicina y Gentamicina. Las posibilidades con los microorganismos no fermentadores son aún más pobres, toda vez que los antibióticos con mejor posibilidad, Amikacina y Sisomicina, apenas inhibieron el $66,6 \%$ de las cepas probadas. 


\section{SUMMARY}

Susceptibility testing on 302 bacterial strains from 17 different species isolated from clinical sources were performed, in order to compare their susceptibility to a new aminoglycoside recently introduced in the country with 10 of the most common antibiotics in clinical use. The usefullness of this type of study when a new type of antibiotic is introduced in a community is emphasized so the clinicians can have a solid knowledge of its activity on the local bacterial strains.

\section{BIBLIOGRAFIA}

1. Baver A.W. et al, Antibiotic susceptibility, by a standar. dized single disc method. Am. J. Clin. Pathol. 1966; 45: 493.

2. Thornsberry Cl, Tlawkins Th. Agar disc diffusion susceptibility testing procedure. CDC Document.

3. Miller G.H.etal. Microbiological studies with Netilm icina. Clin. Trials J. (London). 1980; $17-6: 242$.

4. Szot R, Tabachnick I. Animal studies with Netilmicina. Clin. Trials J. 1980; $17-6: 267$. 